\title{
DETERMINACIÓN DE LA CONCENTRACIÓN LETAL 50 PARA RHIPICEPHALUS (BOOPHILUS) MICROPLUS DE LA FORMAMIDINA (AMITRAZ) EN DIFERENTES POBLACIONES DE LA ARGENTINA
}

\author{
Castelli, M. e. ${ }^{1} ;$ Mangold, A. J. ${ }^{1} ;$ Nava,.$^{1}{ }^{1}$ \& Guglielmone, A. A. ${ }^{1}$
}

\begin{abstract}
RESUMEN
Se determinó la concentración letal 50 (CL 50) para el amitraz (Az) en poblaciones susceptibles de Rhipicephalus (Boophilus) microplus (R. (B) microplus) utilizando tela de nylon (Type 2320, Cerex Advanced Fabrics, Pensacola, FL) impregnados con concentraciones crecientes de Az: 0,0005; $0.0010 ; 0,0020 ; 0.0039 ; 0,0078 ; 0,0156 ; 0,0313 \mu \mathrm{g} / \mathrm{cm} 2$. En ocho poblaciones de $R$. (B) microplus se estimó las CL 50 y los intervalos de confianza del $95 \%$ derivados de la mortalidad de la poblaciones susceptibles de $R$. (B) microplus en relación a la dosis del acaricida, por medio del análisis "probit". El valor de la CL 50 y CL90 y los desvíos estandar correspondientes fueron: 0,00106 $\mu \mathrm{g}$ / $\mathrm{cm} 2 \pm 0,00038$ y $0,00397 \mu \mathrm{g} / \mathrm{cm} 2 \pm 0,00144$, respectivamente. El valor obtenido se utilizó para evaluar la susceptibilidad/resistencia de poblaciones de otras procedencias, probando su eficacia como valor de referencia para monitorear "in vitro" poblaciones de $R$. (B) microplus.
\end{abstract}

Palabras clave: Rhipicephalus (Boophilus) microplus, amitraz, concentración letal 50.

\section{SUMMARY}

\section{Determination of lethal concentration 50 for Rhipicephalus (Boophilus)} microplus of formamidine (amitraz) in different populations of Argentina.

The 50\% lethal concentration (LC 50) of amitraz (Az) for Rhipicephalus (Boophilus) microplus (R. (B) microplus) susceptible population was determined using. Nylon fabric (Type 2320, Ceres Advanced Fabrics Pensacola, FL), impregnated with different concentrations of Az. The following

1.- INTA EEA Rafaela. C.C. 22. (2300) Rafaela, provincia de Santa Fe.

Email: mirtacastelli@wilnet.com.ar

Manuscrito recibido el 25 de octubre de 2012 y aceptado para su publicación el 8 de abril de 2013. 
insecticide concentrations were utilized: $0.0005 ; 0.0010 ; 0.0020 ; 0.0039 ; 0.0078 ; 0.0156 ; 0.0313$ $\mu \mathrm{g} / \mathrm{cm} 2$. The LC 50 was determined for Az in eight susceptible populations of Rhipicephalus (Boophilus) microplus. The LC 50, LC 90 and standard deviation were: $0.00106 \mu \mathrm{g} / \mathrm{cm} 2 \pm 0.00038$ and $0.00397 \mu \mathrm{g} / \mathrm{cm} 2 \pm 0.00144$, respectively. The value obtained was used to evaluate the susceptibility / resistance of populations from other sources, testing its effectiveness as a benchmark for "in vivo" monitoring susceptibility in populations of R. (B) microplus.

Key words: Rhipicephalus (Boophilus) microplus, amitraz, lethal concentration 50.

\section{INTRODUCCIÓN}

Rhipicephalus (Boophilus) microplus (R. (B) microplus), la garrapata común del bovino, ocasiona perjuicios económicos directos e indirectos de gran magnitud a la ganadería, especialmente en zonas tropicales y subtropicales húmedas. Entre ellos se pueden mencionar las pérdidas en la ganancia de peso, de la producción láctea, importantes daños en los cueros que llegan hasta el descarte y los gastos que genera la aplicación de métodos de control (garrapaticidas, mano de obra e infraestructura, entre otros). Además $R$. (B) microplus es el vector de los agentes causales de la babesiosis bovina (Babesia bovis y Babesia bigemina) y está involucrado en la transmisión de Anaplasma marginale, agente causal de la anaplasmosis bovina.

En Argentina se utilizan actualmente varios principios activos para el control de $R$. (B) microplus, como piretroides, formamidinas, avermectinas, fenilpirazol y fluazuron. El 42,5\% de los productores ganaderos usa piretroides, en tanto las formamidinas son aplicadas en un porcentaje menor $(24,7 \%)$. Sólo el 3,7\% emplea avermectinas para el control de las garrapatas; pero se incrementa el porcentaje cuando este principio activo se combina con piretroides $(17,8 \%)$. El método de aplicación más utilizado es el baño de inmersión, el cuál supera en frecuencia de uso a los que emplean la vía tópica o las combinaciones de inyectables con tópicos o con inmersión (Guglielmone et al., 2007). Sin embargo, el amplio uso de los piretroides como método de control se encuentra limitado por la aparición de poblaciones de $R$. (B) microplus resistentes a este principio activo en Corrientes, Santa Fe, Salta, Chaco, Santiago del Estero y norte de Córdoba (Caracostantógolo et al., 1996; Mangold et al., 2000; Mangold et al., 2004). Esta situación devino en la intensificación del uso del amitraz (Az) para los baños de inmersión.

El Az es una formamidina que pertenece a la familia química de las amidinas. Esta droga es más soluble en solventes orgánicos que en agua y se hidroliza fácilmente en medio ácido (Corta et al., 1998; de Villiers et al., 2004).

El Az actúa como agonista de la octopamina tanto a nivel central como periférico, inhibiendo la monoaminooxidasa (MAO). La octopamina es una amina adrenérgica natural de los invertebrados. Se encuentra en el ganglio cerebral y en otros sitios tales como el ganglio cardíaco. El acceso a la hemolinfa la convierte en una posible hormona moduladora de la actividad neuromuscular. En el sistema periférico, el Az puede imitar la acción de la octopamina, cumpliendo una función similar: modula la transmisión neuromuscular actuando por aumento de la liberación del neurotransmisor excitatorio (Evans \& Gee, 1980). Debido 